\title{
Isolation and Characterization of 89K Pathogenicity Island-Positive ST-7 Strains of Streptococcus suis Serotype 2 from Healthy Pigs, Northeast China
}

\author{
Shujie Wang, ${ }^{1}$ Peng Liu, ${ }^{2}$ Chunyu Li, ${ }^{1}$ Yafang Tan, ${ }^{2}$ Xuehui Cai, ${ }^{1}$ \\ Dongsheng Zhou, ${ }^{2}$ and Yongqiang Jiang ${ }^{2}$ \\ ${ }^{1}$ National Key Laboratory of Veterinary Biotechnology, Harbin Veterinary Research Institute, Chinese Academy of Agricultural Science, \\ Harbin 150001, China \\ ${ }^{2}$ State Key Laboratory of Pathogen and Biosecurity, Beijing Institute of Microbiology and Epidemiology, Beijing 100071, China
}

Correspondence should be addressed to Dongsheng Zhou, dongshengzhou1977@gmail.com and

Yongqiang Jiang, jiangyq@nic.bmi.ac.cn

Received 20 February 2012; Accepted 25 March 2012

Academic Editors: M. Dunn, Q. He, and B. Skoldenberg

Copyright (C) 2012 Shujie Wang et al. This is an open access article distributed under the Creative Commons Attribution License, which permits unrestricted use, distribution, and reproduction in any medium, provided the original work is properly cited.

\begin{abstract}
Streptococcus suis is a swine pathogen which can also cause severe infection, such as meningitis, and streptococcal-like toxic shock syndrome (STSS), in humans. In China, most of the S. suis infections in humans were reported in the southern areas with warm and humid climates, but little attention had been paid to the northern areas. Data presented here showed that the virulent serotypes $1,2,7$, and 9 of S. suis could be steadily isolated from the healthy pigs in the pig farms in all the three provinces of Northeast China. Notably, a majority of the serotype 2 isolates belonged to the $89 \mathrm{~K}$ pathogenicity island-positive ST-7 clone that had historically caused the human STSS outbreaks in the Sichuan and Jiangsu provinces of China, although the human STSS case caused by S. suis had never been reported in northern areas of China. Data presented here indicated that the survey of $S$. suis should be expanded to or reinforced in the northern areas of China.
\end{abstract}

\section{Introduction}

Streptococcus suis is an important pathogen that can cause the severe systemic infection in the pigs reported worldwide [1]. S. suis can also be frequently isolated from other animals such as cats, dogs, and horses, and thus it is believed to be a commensal in the animal intestinal flora [2]. A total of 35 serotypes have been characterized for the S. suis isolates from the healthy pigs, but only a limited portion (serotypes 1 to 9 , and 14) of them are responsible for the infections in pigs.

Being a causative agent of a zoonotic disease, S. suis can be transmitted from pigs to humans. Since the first human case caused by S. suis was reported in Denmark, increasing numbers of human cases have been reported in many countries especially including South Asia [3]. Human infections generally can be manifested as meningitis, septicaemia, endocarditis, and deafness. Nearly all the human cases characterized can be ascribed to the handling/consumption of unprocessed pork meat, or to the close contact with pigs [4]. Therefore, most of the infected people are pig farmers, abattoir workers, meat inspectors, butchers, or veterinarian practitioners.

S. suis serotype 2 is the most highly pathogenic one of the 35 serotypes for both pigs and humans [5], and it has caused the two recent outbreaks of human infection in China, which are characterized by a streptococcal toxic shock-like syndrome (STSS), with higher-than-usual human morbidity and mortality [6]. The STSS-causing S. suis serotype 2 strains have acquired a $89 \mathrm{~K}$ pathogenicity island $(89 \mathrm{KPaI})$ harboring multiple virulence determinants [6-8]. The acquisition of $89 \mathrm{KPaI}$ through gene horizontal transfer plays important roles in the rapid adaptation and increased virulence of $S$. suis serotype 2 [6-8].

In the present work, a total of 155 S. suis strains were isolated from the 2204 nose swabs collected from the healthy pigs distributed in all three provinces in Northeast China. At 
least four virulent serotypes, 1, 2, 7, and 9 were discriminated from these strains. Notably, a collection of $89 \mathrm{KPaI}-$ positive ST-7 strains of serotype 2 were identified for the first time in northern areas of China.

\section{Material and Methods}

2.1. Specimens Collection. A total of 2204 nose swabs were collected with the aseptic procedures from 2204 different healthy pigs from March to November, 2007. The pigs included growing, nursery, and finishing ones, and sows from 23 pig farms in Northeast China (Heilongjiang, Liaoning and Jilin provinces).

2.2. Isolation of Bacterial Strains and Genomic DNA. The selective Todd-Hewitt broth containing polymyxin B $(10 \mathrm{mg} / \mathrm{mL})$, nalidixic acid $(15 \mathrm{mg} / \mathrm{mL})$, and crystal violet $(0.1 \mathrm{mg} / \mathrm{mL})$ was used for the primary isolation of S. suis from the nose swab specimens [9]. Bacteria were cultured overnight on the Columbia sheep agar plates at $37^{\circ} \mathrm{C}$ for isolation of the genomic DNA by using a genomic DNA isolation kit (Tigan, Beijing, China).

2.3. PCR Detection. S. suis was identified by detecting a specific $g d h$-amplification product through PCR [10]. The serotypes 1, 2, 7, and 9 were identified by PCR with the previously characterized serotype-specific primers $[11,12]$. PCR was also done to detect the virulence genes $[13,14]$ encoding muramidase-released protein (MRP), extracellular protein factor (EF), and suilysin (SLY) and to detect the SSU05_0943 gene in the 89KPaI [7].

2.4. Multilocus Sequence Typing Analysis (MLST). A previously described MLST scheme [15] was applied to the indicated $S$. suis strains. This MLST scheme involved seven housing-keeping gene loci, that is, cpn60, $d p r, \operatorname{rec} A$, aro $A$, thrA, gki, and mutS, which encoded $60-\mathrm{kDa}$ chaperonin, putative peroxide resistance protein, homologous recombination factor A, 5-enolpyruvylshikimate 3-phosphate synthase, aspartokinase/homoserine dehydrogenase, glucose kinase, and DNA mismatch repair enzyme, respectively. PCR products were purified by using the QIAquick PCR product purification columns and then sequenced from both ends with an ABI Prism 3700 DNA analyzer system. The obtained sequences were compared with the previously defined allelic sequences in the $S$. suis MLST database (http://ssuis.mlst.net/), to identify the allelic profile or sequence type (ST) of each isolate tested herein.

\subsection{Microarray-Based Comparative Genomic Hybridization} (M-CGH). Gene contents were compared between each paired test and reference (05ZYH33) DNAs using a wholegenome DNA microarray $[16,17]$ imprinted with $98 \%$ of the 2194 annotated ORFs of the S. suis serotype 2 strain 05ZYH33 (each ORF was printed in duplicate on a single glass slide). Each paired test and reference (05ZYH33) DNAs were labeled with difference fluorescent dyes (Cy3 or Cy5 dye) and then cohybridized to a microarray slide. Experiments were repeated in duplicate (two biological DNA probes replicates, and accordingly two microarray slides for each strain), for which the incorporated dye was reversed. The hybridized slides were scanned by using a GenePix Personal 4100A Microarray Scanner (Axon Instruments). The scanning images were processed and the data were further analyzed by using GenePix Pro 5.0 software (Axon Instruments) combined with Microsoft Excel software. Spots with signal intensity (median) in the channel of Reference DNA less than two folds of local background intensity (median) were rejected from further analysis. Spots with bad data because of slide abnormalities were discarded as well. Data normalization was performed on the remaining spots by total intensity normalization methods. A ratio of intensity (test DNA normalized intensity/reference DNA normalized intensity) was recorded for each spot and then was converted to $\log _{2}$. Genes with fewer than three data points were considered unreliable and were accordingly removed. The averaged $\log _{2}$ ratio for each remaining gene on the two replicate slides was ultimately calculated. If $20 \%$ of the strains had a gene with missing data, the gene was removed. A $\log _{2}$ value equal to or lower than -1 was taken as defining the absence of a gene in given strain. The binary dataset of absent (0) or present (1) sign genes among strains was displayed by the TreeView software vesion 1.60 [18].

\section{Results and Discussion}

3.1. Identification and Characterization of Total S. suis Strains. The Northeast China region consists of three provinces Jilin, Heilongjiang, and Liaoning. As shown in Figure 1(a), the 2204 nose swab specimens from 2204 different healthy pigs tested could approximately equally assigned into these three provinces. From the 2204 specimens, a total of 155 S. suis strains were isolated (Figure 1(b)), and these isolates were identified as $S$. suis by the positive PCR detection of the S. suis-specific $g d h$ gene [10]. The three provinces Jilin, Heilongjiang, and Liaoning accounted for $83 \%, 12 \%$, and $5 \%$ of the strains isolated, respectively. Accordingly, the S. suis isolation rate (no. of $S$. suis strain isolated/no. of specimens tested) for Jilin, Heilongjiang, and Liaoning were $18.43 \%$, $0.95 \%$, and $2.64 \%$, respectively. The much higher isolation rate for Jilin might be due to the fact that the specimens from Liaoning and Heilongjiang were collected during colder months (in March, April, and October) whereas those from Jilin during warmer months (in June and July).

The serotypes 1, 2, 7, and 9 were screened by PCR for these 155 S. suis isolates with the previously characterized serotype-specific primers of $S$. suis $[11,12]$. Accordingly, the 155 isolates were composed of $39(25 \%)$ serotype-2 strains, $11(7 \%)$ serotype- 9 ones, $7(4 \%)$ serotype-1 ones, 4 (3\%) serotype-7 ones, and $94(61 \%)$ ones of unknown serotypes (Figure 1(c)). The PCR assays showed that all the 39 serotype 2 strains harbored the three virulence genes encoding MRP, EF, and SLY. These results indicated the highly virulent serotype 2 strains could be frequently isolated from the healthy pigs in Northeast China.

3.2. Identification and Characterization of $89 \mathrm{KPaI}$-Positive S. suis Serotype 2. The SSU05_0943 gene in the 89KPaI was 


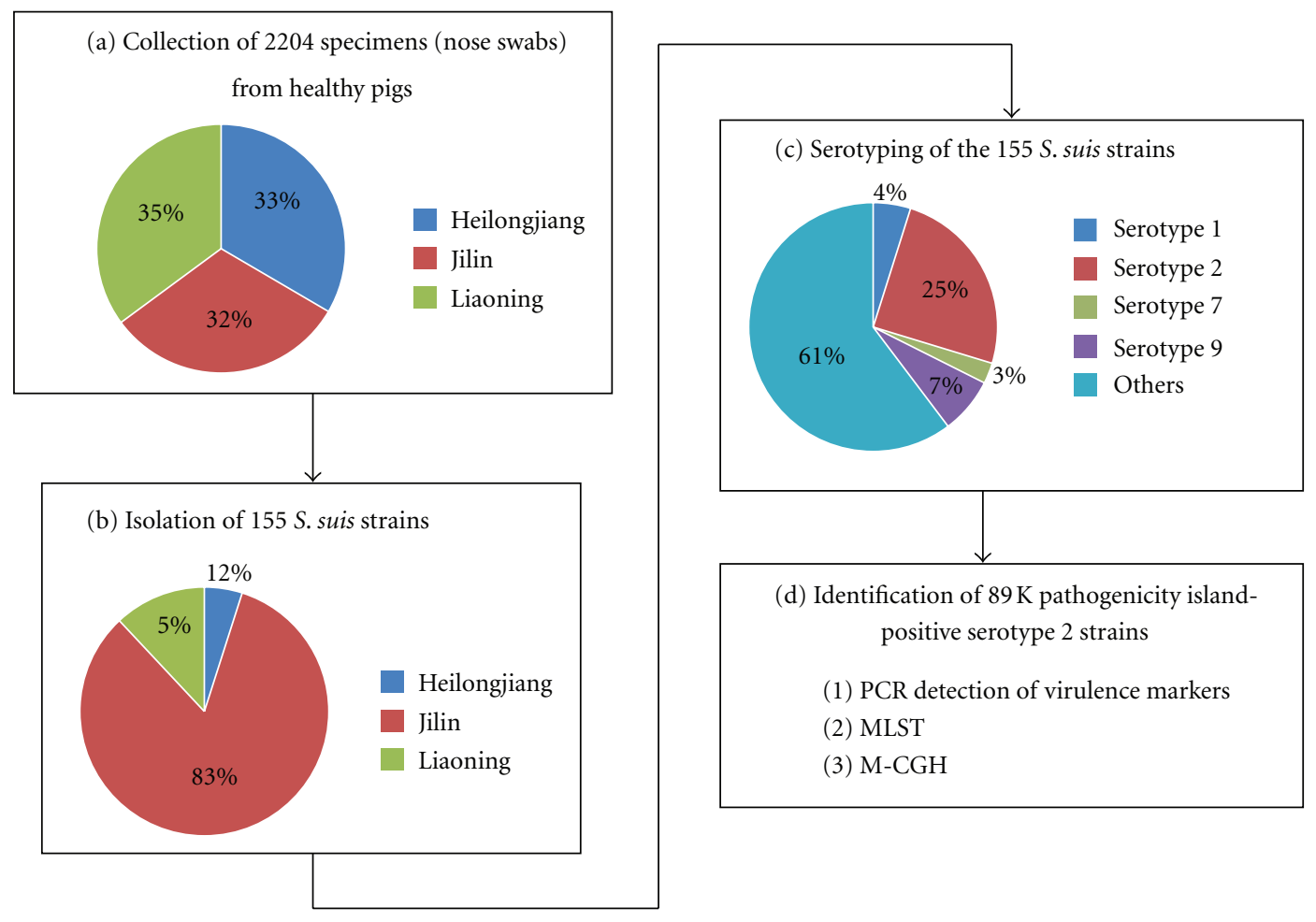

Figure 1: Flow charts of the analyses in this study.

chosen for the PCR-based screening for this island in the 39 serotype 2 strains; 32 (82\%) of these 39 strains gave positive PCR reaction, indicating that the corresponding strains potentially harbored this genomic island.

Of the above 32 strains potentially harboring the $89 \mathrm{KPaI}$, 15 were arbitrarily selected for the MLST assay. All these 15 strains were identified as ST-7 with an allelic profile $1,1,1,1,1,1,3$, which was the same as that of the reference strain 05ZYH33 (an 89KPaI-positive strain, with the determined genome sequence, isolated from the human STSS case [7]). ST-7, represented by the STSS-causing, 89KPaI-positive strains of $S$. suis serotype 2, emerged first in Hong Kong in 1996, and caused 28 cases of human S. suis infection in Jiangsu Province, China, in 1998, and another large outbreak of human infection in Sichuan Province, China, in 2005 [6$8,19]$. As a member of the ST-1 (allelic profile $1,1,1,1,1,1,1$ ) complex, ST-7 is a single-locus variant of ST-1 with increased virulence [19], as demonstrated by the fact that the toxicity of ST-7 to peripheral blood mononuclear cells is greater than that of ST-1 [19].

Of the above 15 ST-7 strains determined by MLST, eight were arbitrarily chosen for the further M-CGH analysis (Figure 2). M-CGH has been established as a standard method for the bacterial comparative genome analysis in our laboratory $[20,21]$. In the present work, a total of S. suis 1918 genes were included in the final microarray dataset, and each gene was categorized as either present (1), absent (0), or missing data for each strain. The eight ST-7 strains tested harbored all the $89 \mathrm{KPaI}$ genes imprinted on the microarray, and they gave the gene profiles almost the same as that of the reference $89 \mathrm{KPaI}-$ positive ST-7 strain 05ZYH33, indicating the high clonal genomic content of 05ZYH33 and the above eight ST-7 strains. Included also in the M-CGH analysis was S735 [22] that was a ST-1 strain $(\mathrm{MRP}+, \mathrm{EF}+, \mathrm{SLY}+$, and $89 \mathrm{KPaI}-$; serotype 2$)$ isolated from a diseased pig in Netherlands in 1963. For S735, the absence of various genome loci (including $89 \mathrm{KPaI}$ ) of $05 \mathrm{ZYH} 33$ was detected by M-CGH. It could be solidly concluded that the above eight strains, characterized by both MLST and M$\mathrm{CGH}$, belonged to the epidemic $89 \mathrm{KPaI}$-positive ST-7 clone of $S$. suis serotype 2 that has historically cause the human STSS outbreaks in China.

3.3. Concluding Remarks. In China, most of the S. suis infections in humans were reported in the southern areas (such as Sichuan, Jiangsu, and Guangdong provinces, and Hongkong) with higher environmental humidity and temperature [23]. However, little attention had been paid to the northern areas of China. Our results showed that the virulent serotypes $1,2,7$, and 9 could be steadily isolated from the healthy pigs in the pig farms in Northeast China. To the best of our knowledge, this is the first report of the isolation of the $89 \mathrm{KPaI}$-positive ST-7 strains of S. suis serotype 2 in Northeast China. Although the human STSS case caused by S. suis has never been reported in northern areas of China, a routine survey of $S$. suis in these geographic regions is needed.

\section{Authors' Contribution}

S. Wang and P. Liu contributed equally to this work. 


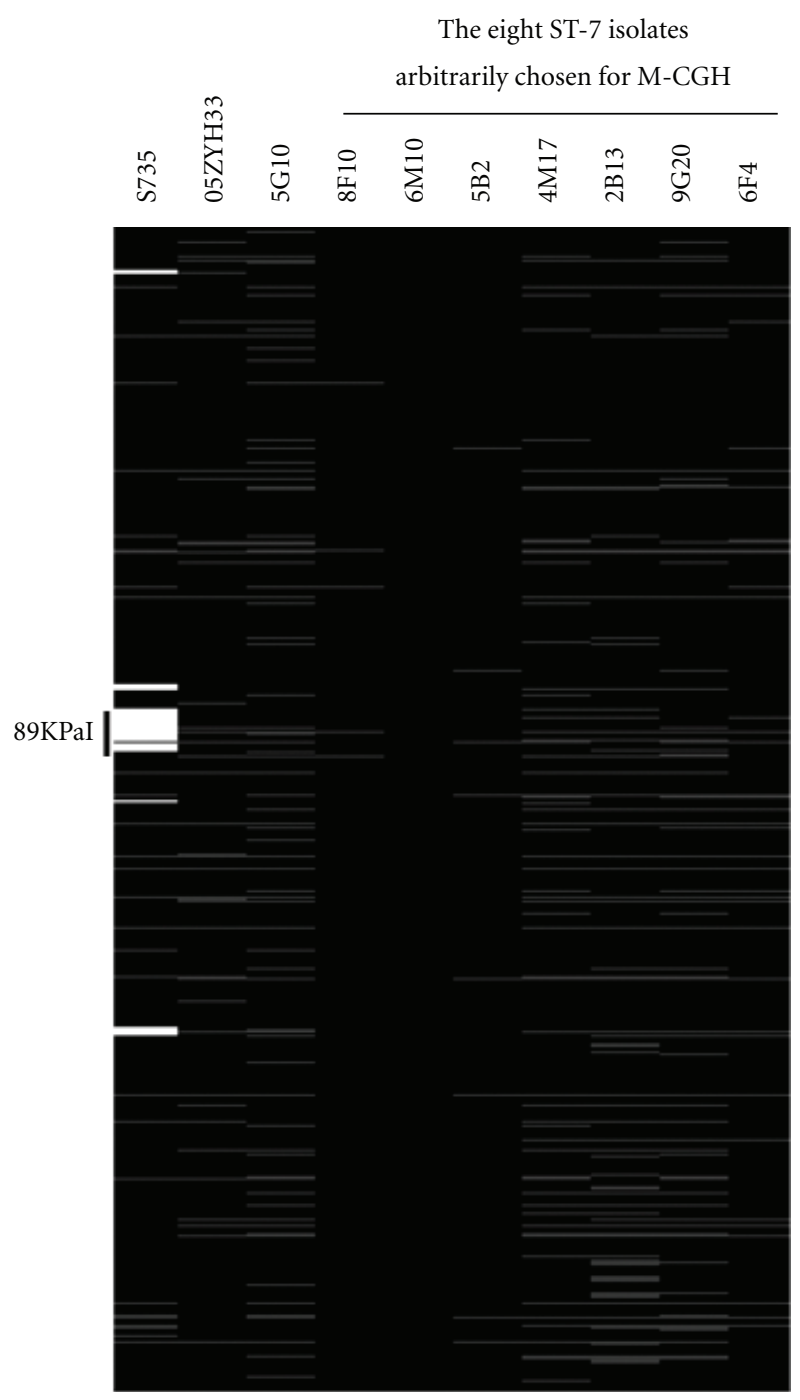

FIgURE 2: Schematic presentation of M-CGH data. Each column represented a strain, while each row standed for a different gene. The strain names were presented on the top. Genes were arranged according to the genomic location of the strain $05 \mathrm{ZYH} 33$. For each individual strain, the presence of a gene was represented by a black box, whereas the absence of a gene corresponded to a white box, and the grey area indicated the missing data.

\section{Acknowledgments}

This work was supported by the National Basic Research Program of China (2012CB518804), the National Natural Science Foundation of China (81171528), and the Fund of the Chinese Ministry of Agriculture Programs (2009ZX08009141B).

\section{References}

[1] M. Segura, "Streptococcus suis an emerging human threat," Journal of Infectious Diseases, vol. 199, no. 1, pp. 4-6, 2009.

[2] J. J. Staats, I. Feder, O. Okwumabua, and M. M. Chengappa, "Streptococcus suis past and present," Veterinary Research Communications, vol. 21, no. 6, pp. 381-407, 1997.

[3] M. Gottschalk, M. Segura, and J. Xu, "Streptococcus suis infections in humans: the Chinese experience and the situation in North America," Animal Health Research Reviews, vol. 8, no. 1, pp. 29-45, 2007.
[4] M. Gottschalk, J. Xu, C. Calzas, and M. Segura, "Streptococcus suis: a new emerging or an old neglected zoonotic pathogen?" Future Microbiology, vol. 5, no. 3, pp. 371-391, 2010.

[5] H. F. L. Wertheim, H. D. T. Nghia, W. Taylor, and C. Schultsz, "Streptococcus suis an emerging human pathogen," Clinical Infectious Diseases, vol. 48, no. 5, pp. 617-625, 2009.

[6] J. Tang, C. Wang, Y. Feng et al., "Streptococcal toxic shock syndrome caused by Streptococcus suis serotype 2," PLoS Medicine, vol. 3, no. 5, article e151, 2006.

[7] C. Chen, J. Tang, W. Dong et al., "A glimpse of streptococcal toxic shock syndrome from comparative genomics of S. suis 2 Chinese isolates," Plos ONE, vol. 2, no. 3, article e315, 2007.

[8] M. Li, C. Wang, Y. Feng et al., "SalK/SalR, a two-component signal transduction system, is essential for full virulence of highly invasive Streptococcus suis serotype 2," PLoS ONE, vol. 3, no. 5, Article ID e2080, 2008.

[9] B. M. Gray, M. A. Pass, and H. C. Dillon, "Laboratory and field evaluation of selective media for isolation of group B streptococci," Journal of Clinical Microbiology, vol. 9, no. 4, pp. 466-470, 1979. 
[10] O. Okwumabua, M. O’Connor, and E. Shull, “A polymerase chain reaction (PCR) assay specific for Streptococcus suis based on the gene encoding the glutamate dehydrogenase," FEMS Microbiology Letters, vol. 218, no. 1, pp. 79-84, 2003.

[11] H. E. Smith, V. Veenbergen, J. van der Velde, M. Damman, H. J. Wisselink, and M. A. Smits, "The cps genes of Streptococcus suis serotypes 1, 2, and 9: development of rapid serotypespecific PCR assays," Journal of Clinical Microbiology, vol. 37, no. 10, pp. 3146-3152, 1999.

[12] H. E. Smith, L. van Bruijnsvoort, H. Buijs, H. J. Wisselink, and M. A. Smits, "Rapid PCR test for Streptococcus suis serotype 7," FEMS Microbiology Letters, vol. 178, no. 2, pp. 265-270, 1999.

[13] H. J. Wisselink, J. J. Joosten, and H. E. Smith, "Multiplex PCR assays for simultaneous detection of six major serotypes and two virulence-associated phenotypes of Streptococcus suis in tonsillar specimens from pigs," Journal of Clinical Microbiology, vol. 40, no. 8, pp. 2922-2929, 2002.

[14] A. G. Allen, S. Bolitho, H. Lindsay et al., "Generation and characterization of a defined mutant of streptococcus suis lacking suilysin," Infection and Immunity, vol. 69, no. 4, pp. 2732-2735, 2001.

[15] S. J. King, J. A. Leigh, P. J. Heath et al., "Development of a multilocus sequence typing scheme for the pig pathogen Streptococcus suis Identification of virulent clones and potential capsular serotype exchange," Journal of Clinical Microbiology, vol. 40, no. 10, pp. 3671-3680, 2002.

[16] X. Zeng, Y. Yuan, Y. Wei et al., "Microarray analysis of temperature-induced transcriptome of Streptococcus suis serotype 2," Vector-Borne and Zoonotic Diseases, vol. 11, no. 3, pp. 215-221, 2011.

[17] Y. Wei, X. Zeng, Y. Yuan et al., "DNA microarray analysis of acid-responsive genes of Streptococcus suis serotype 2," Annals of Microbiology, vol. 61, pp. 505-510, 2010.

[18] M. B. Eisen, P. T. Spellman, P. O. Brown, and D. Botstein, "Cluster analysis and display of genome-wide expression patterns," Proceedings of the National Academy of Sciences of the United States of America, vol. 95, no. 25, pp. 14863-14868, 1998.

[19] C. Ye, X. Zhu, H. Jing et al., "Streptococcus suis sequence type 7 outbreak, Sichuan, China," Emerging Infectious Diseases, vol. 12, no. 8, pp. 1203-1208, 2006.

[20] H. Han, H. C. Wong, B. Kan et al., "Genome plasticity of Vibrio parahaemolyticus: microevolution of the "pandemic group"'” BMC Genomics, vol. 9, article 570, 2008.

[21] D. Zhou, Y. Han, Y. Song et al., "DNA microarray analysis of genome dynamics in Yersinia pestis: insights into bacterial genome microevolution and niche adaptation," Journal of Bacteriology, vol. 186, no. 15, pp. 5138-5146, 2004.

[22] N. Fittipaldi, J. Harel, B. D’Amours, S. Lacouture, M. Kobisch, and M. Gottschalk, "Potential use of an unencapsulated and aromatic amino acid-auxotrophic Streptococcus suis mutant as a live attenuated vaccine in swine," Vaccine, vol. 25, no. 18, pp. 3524-3535, 2007.

[23] Y. Feng, H. Zhang, Y. Ma, and G. F. Gao, "Uncovering newly emerging variants of Streptococcus suis, an important zoonotic agent," Trends in Microbiology, vol. 18, no. 3, pp. 124$131,2010$. 

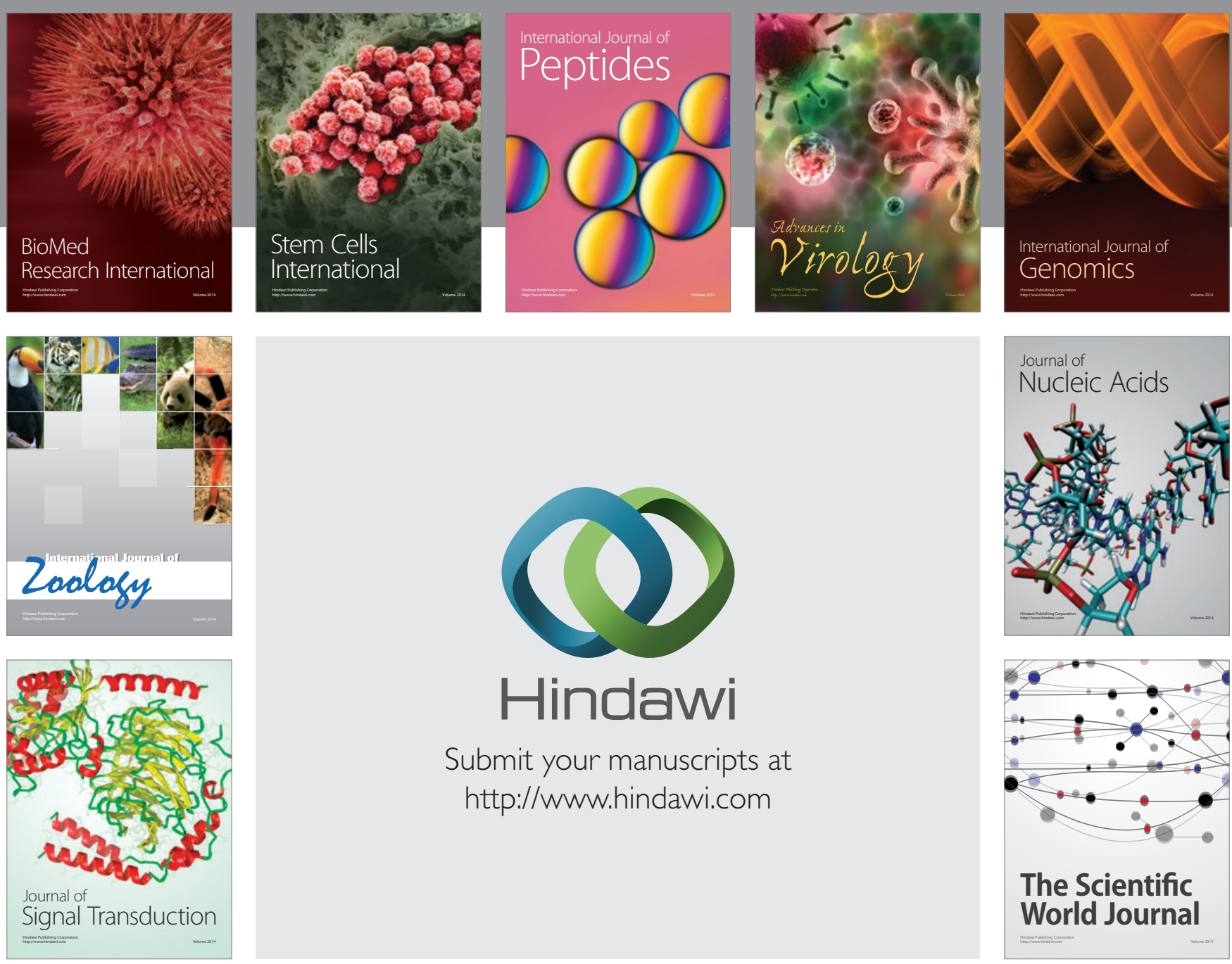

Submit your manuscripts at

http://www.hindawi.com
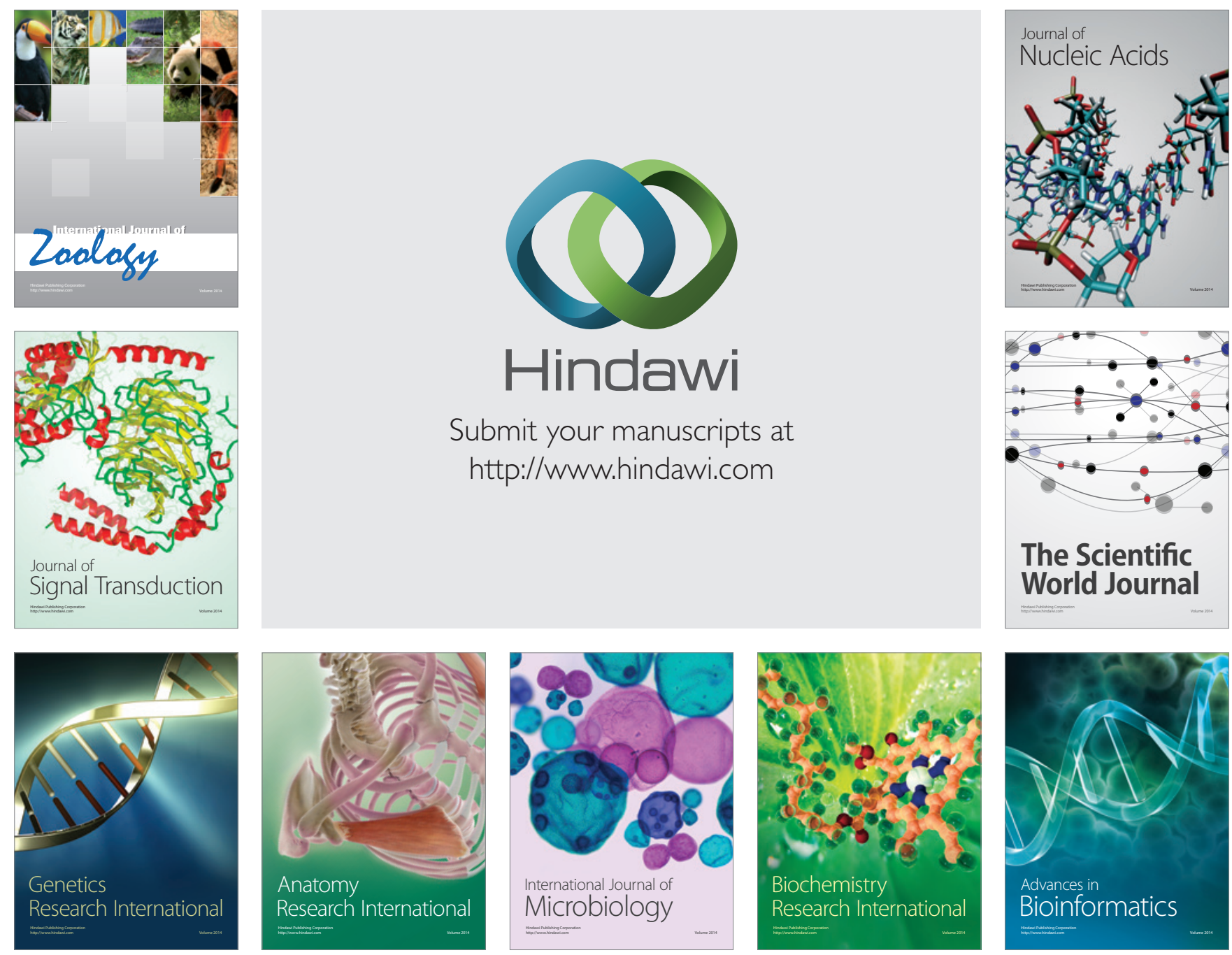

The Scientific World Journal
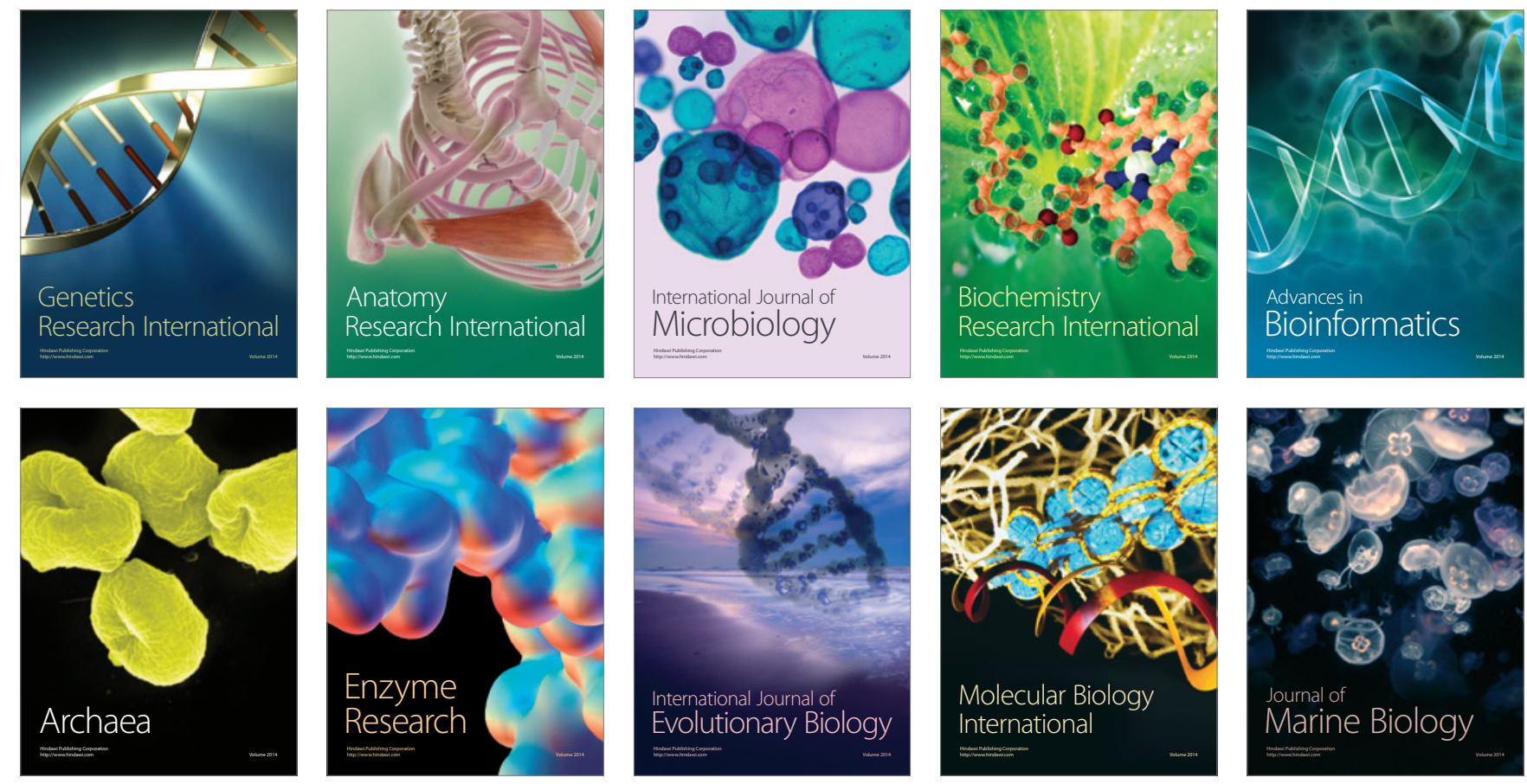